\title{
THE METHOD TO SOLVE DRIFT IN MAIN GEOMAGNETIC FIELD
}

\author{
Gendenpuntsag Bayanjargal \\ Institute of Astronomy and Geophysics, Mongolian Academy of Sciences, Ulaanbaatar, Mongolia \\ E-mail: gbayan27@yahoo.com
}

\begin{abstract}
The method to solve the velocity of drift in MGF is written in this paper. This method is based on the dynamo theory for geomagnetic field. The velocity of drift in the main geomagnetic field is also solved from the condition that the power variation of residual field equals zero in few years.
\end{abstract}

Keywords: Drift of geomagnetic field, dynamo theory for geomagnetic field

\section{INTRODUCTION}

Drift in main geomagnetic field has been studying since it is discovered by Edmund Halley in 1692. When E. Halley reconstructs map of geomagnetic field of the Earth, he observed drifts in lines with equal values of the main geomagnetic field from old maps produced in different years. He studied the drifts diligently and he obtained that mean value of the drifts was -0.5 degree for whole Earth in year. Halley's method is very easy. $\mathrm{He}$ measured the drifts of lines with equal values on the old maps of main geomagnetic field. Then he obtained the average of values measured. This Halley's method is being used today. Unfortunately, this Halley's method for the drift in the main geomagnetic field is not developed.[3]

Gauss C.F included the method of spherical harmonic potential to the geomagnetism from the potential theory in 1839. This method obtains the scalar potential for geomagnetic field form the Laplace's equation written for scalar potential of geomagnetic field. And values of the Gaussian coefficients in the scalar potential for geomagnetic field are detected by data measured in satellites and observatories. IGRF has also updating the
Gaussian coefficients every 5 years since 1900 .

Generally, the drift in geomagnetic field is being studied by the method based on the scalar potential for geomagnetic field. I think this method is not the best advanced method for the drift in geomagnetic field.

Strong field sourced at Earth center spreads inner core, liquid core, mantle and crust and it is observed as geomagnetic field on Earth surface.

Phenomenon,which the drift in the geomagnetic field is observed about Earth's surface, is caused by fluid motions with electrical conductivity on Core Mantle Boundary (CMB).

Interaction between the strong field and fluid motions with electrical conductivity on $\mathrm{CMB}$ is expressed by induction equation. Form of the equation, which can study the drift in geomagnetic field about Earth surface, must have been detected.

We have obtained the form of equation about Earth surface similar to the properties of the induction equation on CMB. And Yukutake, $\mathrm{T}$ wrote about the form of equation in 1962,[6]. We can also solve the drift in geomagnetic field from the equation written 
about the Earth surface.

The purpose of this paper is to write the above method for researchers in easy form.

\section{THE FORM OF INDUCTION EQUATION ABOUT EARTH'S SURFACE}

Strong field is sourced by the dynamic processes in sub-shell of georeactor at the center of the Earth's inner core. [4] The strong field spreads according to inner core, liquid core, mantle and crust. And it is observed as the main geomagnetic field.

Enough intensity motions are not inner core and mantle. Therefore inner core and mantle can affect only by the diffusing in the spreading of field.

Intensity motions of conductivity liquid also exist in the liquid core. The motions can interact by the induction with the strong field.

The scale of radial component of the fluid motions in the liquid core is many times smaller than the scale of horizontal component. The reason the liquid core is in the incompressible and unexpanded condition. And the density at bottom of liquid core is $23 \%$ more than the density of upper part of liquid core.[4] This density difference in the liquid core blocks the possibility that the fluid motions are sourced by the thermal convection in the whole liquid core. Therefore the scale of radial component of the fluid motions in the liquid core is small.

The most effectual fluid motions in the liquid core exist about the CMB. The fluid motions on $\mathrm{CMB}$ are horizontal motions. Its radial component is also small and it is in the turbulent form.

We can write the governing differential equation of interaction between the fluid motions and the strong field on CMB. It is written as

$$
\frac{\partial \vec{B}}{\partial t}=\vec{\nabla} \times(\vec{V} \times \vec{B})+\eta \nabla^{2} \vec{B} .
$$

The Eq.(1) is also called the induction equation. Here $\eta$ is the coefficient of magnetic diffusivity and $\vec{V}$ is the local velocity of the conductive fluid motions on the CMB.
It is possibility that the fluid motions and the field on the $\mathrm{CMB}$ are divided into two components such as mean motions and turbulent motions. They are written in the form

$$
\left.\begin{array}{l}
\vec{V}=\vec{V}_{0}+\vec{v}=\text { mean }+ \text { fluctuations } \\
\vec{B}=\vec{B}_{0}+\vec{b}=\text { mean }+ \text { fluctuations }
\end{array}\right\}
$$

Velocity of the mean motions is slow varied and its $L$-scale is long. $l$-scale of the turbulent motions is small as $L>>l$. The velocity of the turbulent motions rapidly changes and it has fluctuations. If the conditions in the Eq. (2) and $L>>l$ the ratio in its scales are fulfilled on $\mathrm{CMB}$, the dynamo theory for mean field could also used in the Eq.(1).

The westward drift is a phenomenon observed in main geomagnetic field on the Earth's surface in the large interval of space and time. And the possibility, which the effects with small interval of space and time on CMB are being observed on the Earth's surface, is small. Thus the dynamo theory for mean field has been used for the approaching in the induction equation.

Therefore the Eq. (1) must be averaged as by Reynold's rules for averaging when the Eq.(2) is substituted into it. It can be written in the form 


$$
\left\langle\frac{\partial \vec{B}_{0}}{\partial t}+\frac{\partial \vec{b}}{\partial t}\right\rangle=\vec{\nabla} \times\left\langle\left(\vec{V}_{0}+\vec{v}\right) \times\left(\vec{B}_{0}+\vec{b}\right)\right\rangle+\eta \nabla^{2}\left\langle\vec{B}_{0}+\vec{b}\right\rangle .
$$

The Eq.(3) becomes as

$$
\frac{\partial \vec{B}_{0}}{\partial t}=\vec{\nabla} \times\left(\vec{V}_{0} \times \vec{B}_{0}\right)+\vec{\nabla} \times\langle\vec{v} \times \vec{b}\rangle+\eta \nabla^{2} \vec{B}_{0} .
$$

Where $\vec{\varepsilon}=\langle\vec{v} \times \vec{b}\rangle$ is called the mean electromotive force. There is a linear relation between the mean electromotive force $\overrightarrow{\mathcal{E}}$ and

$$
\vec{\varepsilon}=\alpha \vec{B}_{0}-\beta \vec{\nabla} \times \vec{B}_{0}+\ldots, \quad \alpha=-\frac{1}{3}\langle\vec{v} \cdot(\vec{\nabla} \times \vec{v})\rangle \tau \quad \text { and } \beta=\frac{1}{3} v^{2} \tau
$$

They are called Taylor's expansion. Here $\alpha$ is the coefficient of the helical effects of fluid motion on the $\mathrm{CMB}, \boldsymbol{\beta}$ is the coefficient for the turbulent diffusivity and $\tau$ is life-time of the helixes.

When the Eq. (5) is substituted into the Eq. (4), it could be rewritten in the following form

$$
\frac{\partial \vec{B}_{0}}{\partial t}+\left(\vec{V}_{0} \cdot \vec{\nabla}\right) \vec{B}_{0}=\left(\vec{B}_{0} \cdot \vec{\nabla}\right) \vec{V}_{0}-\vec{B}_{0}\left(\vec{\nabla} \cdot \vec{V}_{0}\right)+\vec{\nabla} \times \alpha \vec{B}_{0}+(\eta+\beta) \nabla^{2} \vec{B}_{0}
$$

where $\left(\vec{V}_{0} \cdot \vec{\nabla}\right) \vec{B}_{0}$ is advection term, $\left(\vec{B}_{0} \cdot \vec{\nabla}\right) \vec{V}_{0}$ is stretching term, $-\vec{B}_{0}\left(\vec{\nabla} \cdot \vec{V}_{0}\right)$ is compression term, $(\eta+\beta) \nabla^{2} \vec{B}_{0}$ is diffusion and decay term. $\vec{V}_{0}$ is the mean velocity of the conductivity of the fluid motion on the CMB.

The core flow is usually assumed that it exists in the incompressible and unexpanded condition. Thus the stretching term $\left(\left(\vec{B}_{0} \cdot \vec{\nabla}\right) \vec{V}_{0}\right.$ ) and the compression term $\left(-\vec{B}_{0}(\vec{\nabla} \cdot \vec{V})_{0}\right)$ in the $\mathrm{Eq}(6)$ are vanished on the CMB. And the terms $\vec{\nabla} \times \alpha \vec{B}_{0}$ and $\beta \nabla^{2} \vec{B}_{0}$ in the Eq.(6) show turbulent effects in the fluid motions on the CMB. The scales and the life-time $\tau$ of $\alpha$ and $\beta$ in the Eq.(6) are small. Therefore the

$$
\frac{\partial \vec{B}}{\partial t}+\left(\vec{v}^{\prime}{ }_{0} \cdot \vec{\nabla}\right) \vec{B}=\vec{R}(\vec{r}, t)
$$

where $\vec{r}$ denotes the positional vector and $\vec{v}_{0}^{\prime}$ is the drift velocity of the field on the Earth's effects of the terms may be observed around one point or one observatory on the Earth's surface during short time. But the effects of the terms with $\alpha$ and $\beta$ is small in the large interval of space and time. Thus the influence of the terms with $\alpha$ and $\beta$ is very small in the same equation as $\mathrm{Eq}(6)$ that will be written for the surface of the Earth or in the mantle.

Above $\mathrm{Eq}(6)$ is only valid for the liquid core. But it can be seen from the Eq.(6) that the advection term is the main factor in the secular variation of MGF on the Earth's surface.

We can write an equation of the same kind with the Eq.(6) on the Earth's surface. The equation is

surface or in the mantle. $\vec{R}(\vec{r}, t)$ is the residual field after the westward drift has been 
subtracted from the observed time-variation. $\vec{R}(\vec{r}, t)$ can be also described as the right hand side of the Eq.(6) but the influence of these terms is decreased by mantle. And the effects of these terms on the residual field in the Eq.(7) are observed as weaker than those in the Eq.(6) on the Earth's surface.

$$
P=\int[\vec{R}(\vec{r}, t)]^{2} d s
$$

where $d s$ is an interval of space and time, $\vec{R}(\vec{r}, t)$ is a residual field, $[\vec{R}(\vec{r}, t)]^{2}$ is a variance of the power density of the residual field in the small time interval and $P$ is the power of the residual field.

The power of the residual field weakly depends on the same terms as the right hand

$$
\delta P=0 \text {. }
$$

Once the field distribution $\vec{B}$ and its time derivative $\partial \vec{B} / \partial t$ are known, we would be able to find the velocity $\vec{v}_{0}^{\prime}$ from the condition that the power variation of the residual field
Therefore it is seen from the Eqs.(6-7) that the variation of MGF on the Earth's surface depends on the mean velocity of the conductivity of the fluid motion on the CMB. Now, the total power of the residual field can be rewrite in the form

\section{To solve velocity}

The power variation of the residual field in the $\mathrm{Eq}(10)$ could be rewritten in the form as

$$
\delta P(t, r, \theta, \varphi, \dot{\theta}, \omega)=\delta \int R^{2}(t, r, \theta, \varphi, \omega, \dot{\theta}) d s=\delta \int\left(R_{x}^{2}+R_{y}^{2}+R_{z}^{2}\right) d s=0 .
$$

side of the Eq.(6). The power variation of the strong toroidal field sourced in sub-shell in the Earth's inner core is also very small and it equals to zero in a time of 20 years.

Therefore the power variation of the residual field equals to zero in a time of 20 years as

equals to zero on the Eq.(9).

In this paper, the drift velocity $\vec{v}_{0}^{\prime}$ of field has been solved from the condition in the Eq. (9).

The following equation as

$R_{x} \cdot \frac{\partial R_{x}}{\partial \omega} \delta \omega+R_{x} \cdot \frac{\partial R_{x}}{\partial \dot{\theta}} \delta \dot{\theta}+R_{y} \cdot \frac{\partial R_{y}}{\partial \omega} \delta \omega+R_{y} \cdot \frac{\partial R_{y}}{\partial \dot{\theta}} \delta \dot{\theta}+R_{z} \cdot \frac{\partial R_{z}}{\partial \omega} \delta \omega+R_{z} \cdot \frac{\partial R_{z}}{\partial \dot{\theta}} \delta \dot{\theta}=0$

could be written from the condition of variation in the Eq. (10).

The Eq. (11) could be also rewritten in the form as

$$
\left.\begin{array}{l}
R_{x} \cdot \frac{\partial R_{x}}{\partial \omega}+R_{y} \cdot \frac{\partial R_{y}}{\partial \omega}+R_{z} \cdot \frac{\partial R_{z}}{\partial \omega}=0 \\
R_{x} \cdot \frac{\partial R_{x}}{\partial \dot{\theta}}+R_{y} \cdot \frac{\partial R_{z}}{\partial \dot{\theta}}+R_{z} \cdot \frac{\partial R_{z}}{\partial \dot{\theta}}=0
\end{array}\right\} .
$$




$$
\left.\begin{array}{l}
\dot{\theta} \cdot M_{1}(t, r, \theta, \varphi)+\omega \cdot P_{1}(t, r, \theta, \varphi)=f_{1}(t, r, \theta, \varphi) \\
\dot{\theta} \cdot M_{2}(t, r, \theta, \varphi)+\omega \cdot P_{2}(t, r, \theta, \varphi)=f_{2}(t, r, \theta, \varphi)
\end{array}\right\}
$$

Where $M_{1}(t, r, \theta, \varphi), \quad P_{1}(t, r, \theta, \varphi), \quad$ The distribution functions of angular $f_{1}(t, r, \theta, \varphi), M_{2}(t, r, \theta, \varphi), P_{2}(t, r, \theta, \varphi)$ and $f_{2}(t, r, \theta, \varphi)$ are analytic functions depended on $t, r, \theta$ and $\varphi$.

velocities of field drift $\dot{\theta}(t, r, \theta, \varphi)$ and

$\omega(t, r, \theta, \varphi)_{\text {could be solved in the form as }}$

$$
\left.\begin{array}{l}
\dot{\theta}(t, r, \theta, \varphi)=\frac{f_{1}(t, r, \theta, \varphi) \cdot P_{2}(t, r, \theta, \varphi)-f_{2}(t, r, \theta, \varphi) \cdot P_{1}(t, r, \theta, \varphi)}{M_{1}(t, r, \theta, \varphi) \cdot P_{2}(t, r, \theta, \varphi)-M_{2}(t, r, \theta, \varphi) \cdot P_{1}(t, r, \theta, \varphi)} \\
\omega(t, r, \theta, \varphi)=\frac{M_{1}(t, r, \theta, \varphi) \cdot f_{2}(t, r, \theta, \varphi)-M_{2}(t, r, \theta, \varphi) \cdot f_{1}(t, r, \theta, \varphi)}{M_{1}(t, r, \theta, \varphi) \cdot P_{2}(t, r, \theta, \varphi)-M_{2}(t, r, \theta, \varphi) \cdot P_{1}(t, r, \theta, \varphi)}
\end{array}\right\}
$$

from the system of equations in the $\mathrm{Eq}(13)$.

field drift can be solved how they are averaged

The average values of angular velocities of by the space and time as

$$
\dot{\theta} \approx \frac{\int \dot{\theta}(t, r, \theta, \varphi) d s}{\int d s} \text { and } \omega \approx \frac{\int \omega(t, r, \theta, \varphi) d s}{\int d s} .
$$

If integrals in the Eq. (15) are difficult to integrate, the easy polynomial forms as the Eq.
(16) could be also built by the values solved via the Eq. (14).

\section{AN EXAMPLE}

On the Europe, we have obtained main geomagnetic field such as distribution functions of space and time for

$B_{x}=C_{00}+C_{01} \varphi+C_{02} \varphi^{2}+C_{03} \varphi^{3}+C_{10} \theta+C_{11} \theta \varphi+C_{12} \theta \varphi^{2}+C_{20} \theta^{2}+C_{21} \varphi \theta^{2}+C_{30} \theta^{3}$

where $\varphi$ is longitude, $\theta$ is latitude, and $\mathrm{C}_{00}$ ,$\ldots \mathrm{C}_{30}$ are polynomial coefficients. The dependences on time in the polynomial coefficients for components $B_{X}, B_{Y}$, and $B_{Z}$, are shown on the table 1.

The functions as the Eq. (16) are also built by the values of main geomagnetic field abstracted from data of observatories located on the Europe. Possibility levels of the functions as the Eq. (16) are about 97-99 percent by the reduced chi-square test.

The functions as the Eq. (16) are also obtained in the NED system of coordinate but the velocity of field drift is solved in the coordinate system of ECEF. Therefore the distribution functions in the Eq. (16) for NED must be transformed to ECEF but the quadrate of a vector is not varied by the inversion operation or the rotation of coordinate system on the Earth's surface. It is expressed as

$$
\left[\vec{R}_{e}(\vec{r}, t)\right]^{2}=\left[\hat{I} \frac{\partial \vec{B}}{\partial t}+\hat{I}\left(\vec{v}^{\prime}{ }_{0} \cdot \vec{\nabla}_{e}\right) \vec{B}\right]^{2}=\hat{I}^{2}\left[\frac{\partial \vec{B}}{\partial t}+\left(\vec{v}^{\prime}{ }_{0} \cdot \vec{\nabla}_{e}\right) \vec{B}\right]^{2}=\left[\frac{\partial \vec{B}}{\partial t}+\left(\vec{v}^{\prime}{ }_{0} \cdot \vec{\nabla}_{e}\right) \vec{B}\right]^{2}
$$


where $\hat{I}$-is the inversion operator from NED to ECEF, $\vec{\nabla}_{e}$-is Nable's operator in ECEF. Thus

we can write components of the residual field as

$$
R_{e x}(\vec{r}, t)=\frac{\partial B_{x}}{\partial t}+\left[v_{0 x}^{\prime} \frac{\partial B_{x}}{\partial x}+v^{\prime}{ }_{0 y}^{\frac{\partial B_{x}}{\partial y}}+v^{\prime} \quad \frac{\partial B_{x}}{\partial z}\right] .
$$

Table 1 .

\begin{tabular}{|c|c|c|c|}
\hline \multirow[b]{3}{*}{$\mathrm{C}_{00}$} & \multicolumn{3}{|c|}{ Europe } \\
\hline & $\mathrm{B}_{\mathrm{x}}$ & $\mathrm{B}_{\mathrm{y}}$ & $\mathrm{B}_{\mathrm{z}}$ \\
\hline & $-0.79 t^{3}+28.22 t^{2}-253.34 t+49320.94$ & $-0.601 t^{3}+24.32 t^{2}-269.764 t+1814.07$ & $-2.81 t^{3}+86.55 t^{2}-730.61 t-42252.38$ \\
\hline $\mathrm{C}_{01}$ & $-290.164 t+6472.407$ & $-1.122 \mathrm{t}^{3+} 32.23 \mathrm{t}^{2}-162.8 \mathrm{t}+4613.3$ & $-4.596 \mathrm{t}^{3}+140.107 \mathrm{t}^{2}-833.83 \mathrm{t}-1043.21$ \\
\hline $\mathrm{C}_{02}$ & $212.34 \mathrm{t}+5017.157$ & $-6.53 t^{2}+315.95 t-16129.51$ & $33.78 \mathrm{t}^{2}-1224.01 \mathrm{t}+15682.71$ \\
\hline $\mathrm{C}_{03}$ & $-0.048 t^{3}-1.42 t^{2}+34.98 t-681.798$ & $-2.48 t^{2}+96.67 t+655.054$ & $-5.79 t^{2}+148.61 t-3773.10$ \\
\hline $\mathrm{C}_{10}$ & $2.611 t^{3}-91.474 t^{2}+922.32 t-38396.769$ & $2.22 t^{3}-85.49 t^{2}+1037.27 t-7733.42$ & $9.59 t^{3}-308.56 t^{2}+2816.7 t+192757.05$ \\
\hline $\mathrm{C}_{11}$ & $453.386 \mathrm{t}-16664.086$ & $-446.87 t+11705.44$ & $9.24 t^{3}-298.33 t^{2}+2267.3 t+8822.88$ \\
\hline $\mathrm{C}_{12}$ & $-191.376 \mathrm{t}-6551.478$ & $-228.86 t+12840.865$ & $-1.88 \mathrm{t}^{3}+16.5 \mathrm{t}^{2}+777.27 \mathrm{t}-7568.86$ \\
\hline $\mathrm{C}_{20}$ & $-2.751 t^{3}+95.57 t^{2}-1006.358 t+5209.597$ & $-2.72 t^{3}+98.78 t^{2}-1096.7 t+5678.08$ & $-10.76 \mathrm{t}^{3}+357.11 \mathrm{t}^{2}-3361.87 \mathrm{t}-142531.1$ \\
\hline $\mathrm{C}_{21}$ & $-200.73 t+10926.704$ & $304.28 \mathrm{t}-8005.95$ & $-4.55 t^{3}+156.06 t^{2}-1390.52 t-7658.87$ \\
\hline $\mathrm{C}_{30}$ & $0.945 t^{3}-32.644 t^{2}+349.745 t+73.998$ & $1.059 \mathrm{t}^{3}-36.48 \mathrm{t}^{2}+366.02 \mathrm{t}-1303.48$ & $3.91 t^{3}-132.97 t^{2}+1283.62 t+38877.98$ \\
\hline
\end{tabular}

And the derivatives in the Eq. (18) as $\frac{\partial B_{x}}{\partial x}=\frac{\partial B_{x}}{\partial \varphi} \frac{\partial \varphi}{\partial x}+\frac{\partial B_{x}}{\partial \theta} \frac{\partial \theta}{\partial x}$ could be solved by the Eq.

(16) and the easy trigonometric ratios as

$$
\begin{gathered}
\frac{\partial \varphi}{\partial x}=\frac{\partial}{\partial x} \arccos \left(\frac{x}{\sqrt{x^{2}+y^{2}}}\right)=-\frac{\sin \varphi}{r \cos \theta}, \frac{\partial \theta}{\partial x}=-\frac{\cos \varphi \sin \theta}{r}, \frac{\partial \varphi}{\partial y}=\frac{\cos \varphi}{r \cos \theta}, \frac{\partial \varphi}{\partial z}=0, \\
\frac{\partial \theta}{\partial x}=-\frac{\cos \varphi \sin \theta}{r}, \frac{\partial \theta}{d y}=-\frac{\sin \varphi \sin \theta}{r}, \text { and } \frac{\partial \theta}{\partial z}=\frac{\cos \theta}{r} .
\end{gathered}
$$

The components of velocity are expanded as

$$
v_{0 x}^{\prime}=-r \sin \theta \cos \varphi \cdot \dot{\theta}-r \cos \theta \sin \varphi \cdot \omega .
$$

The Eq.(18) and Eq.(20) must be substituted into the Eq. (12). Then the form as the Eq. (13) is obtained from the Eq. (12). And the distribution functions of angular velocities of

$$
\dot{\theta}_{e u} \approx \frac{\int_{1991}^{2006}\left[\int_{0}^{R_{0}} \int_{\pi / 6}^{4 \pi / 9} \int_{-\pi / 18}^{2 \pi / 9} \dot{\theta}_{e u}(t, r, \theta, \varphi) \cdot r^{2} \cos \theta d \varphi d \theta d r d t\right.}{\int_{1991}^{2006}\left[\int_{0}^{R_{0}} 4 \pi / 9 \int_{\pi / 6}^{4 \pi / 9} \int^{2 \pi / 18} r^{2} \cos \theta d \varphi d \theta d r d t\right.} \approx-0.02 \operatorname{deg} \text { ree / year }
$$


and

$$
\begin{aligned}
& 2006 R_{0} 4 \pi / 92 \pi / 9 \\
& \int_{991}^{2006}\left[\int_{0}^{R} \int_{\pi / 6}^{9} \int_{10 \pi}^{9} \omega_{e u}(t, r, \theta, \varphi) \cdot r^{2} \cos \theta d \varphi d \theta d r d t\right. \\
& \omega_{\text {eu }} \approx \frac{19910 \pi / 6 \pi / 9}{2006 R_{0} 4 \pi / 92 \pi / 9} \approx-0.303 \mathrm{deg} \text { ree / year } . \\
& \int\left[\iint_{1}^{20} r^{2} \cos \theta d \varphi d \theta d r d t\right. \\
& 19910 \pi / 6-\pi / 18
\end{aligned}
$$

\section{CONCLUSIONS}

The advection term $\left(\vec{V}_{0} \cdot \vec{\nabla}\right) \vec{B}_{0}$ in the Eq. (6) is the main factor in the time variation or the secular variation of MGF. In other words, the Eq. (6) for the CMB very weakly depends on the terms in the right hand side. And the variation does not exist in the power of the strong field in a few years. These are the theoretical basis of this method to define the drift in geomagnetic field.

We could predict that the power of MGF

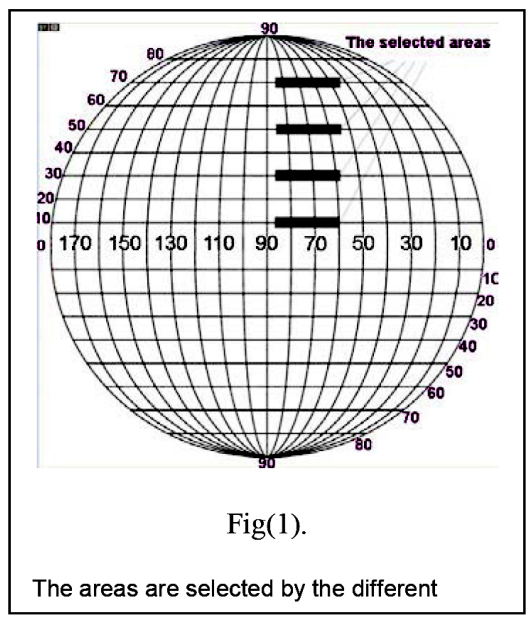

for whole Earth is steady in few years and the variation in MGF on points of Earth's surface is sourced by the movement of field.

If any latitudinal dependence exists in the drift of geomagnetic field, it must be detected clearly by this method on the selected areas in the fig (1). And some models for MGF as shown on the fig (2) could be tested by this latitudinal dependence on the drift of geomagnetic field.

\section{REFERENCES}

1. Bullard, E.C., Freedman, C., Gellman, H., and Nexon, J., The westward drift of the Earth's magnetic field. Philosophical Transactions of the Royal Society A, 43, 67-92, 1950.

2. Bloxham, J., and A. Jackson, Fluid flow near the surface of the Earth's outer core, Rev. Geophys., 29(1), 97-120, 1991.

3. Halley, E., On the cause of the change in the variation of the magnetic needle, with an hypothesis of the structure of the internal parts of the earth. Philosophical Transactions of the Royal Society of London, 17, 470-478, 1692. 
4. Herndon, J.M., Uniqueness of Herndon's georeactor: Energy source and production mechanism for Earth's magnetic field, 2009. arXiv.org/abs/0901.4509

5. Holme, R., Large-Scale Flow in the Core. Treatise on geophysics volume 8. Elsevier, 107-128, 2007.

6. Yukutake, T., The westward drift of the magnetic field of the Earth. Bulletin of the Earthquake Research Institute, 40, 1-65, 1962. 